ARTICLE

https://doi.org/10.1038/s41467-020-14346-5

\title{
Ice $I_{c}$ without stacking disorder by evacuating hydrogen from hydrogen hydrate
}

\author{
Kazuki Komatsu (10 ${ }^{1 \star}$, Shinichi Machida ${ }^{2}$, Fumiya Noritake ${ }^{3,4}$, Takanori Hattori ${ }^{5}$, Asami Sano-Furukawa ${ }^{5}$, \\ Ryo Yamane ${ }^{1}$, Keishiro Yamashita ${ }^{1} \&$ Hiroyuki Kagi ${ }^{1}$
}

Water freezes below $0^{\circ} \mathrm{C}$ at ambient pressure ordinarily to ice $I_{h}$, with hexagonal stacking sequence. Under certain conditions, ice with a cubic stacking sequence can also be formed, but ideal ice $I_{c}$ without stacking-disorder has never been formed until recently. Here we demonstrate a route to obtain ice $I_{c}$ without stacking-disorder by degassing hydrogen from the high-pressure form of hydrogen hydrate, $C_{2}$, which has a host framework isostructural with ice $I_{c}$. The stacking-disorder free ice $I_{c}$ is formed from $C_{2}$ via an intermediate amorphous or nano-crystalline form under decompression, unlike the direct transformations occurring in ice XVI from neon hydrate, or ice XVII from hydrogen hydrate. The obtained ice $I_{c}$ shows remarkable thermal stability, until the phase transition to ice $I_{h}$ at $250 \mathrm{~K}$, originating from the lack of dislocations. This discovery of ideal ice $\mathrm{I}_{\mathrm{c}}$ will promote understanding of the role of stacking-disorder on the physical properties of ice as a counter end-member of ice $I_{h}$.

\footnotetext{
${ }^{1}$ Geochemical Research Center, Graduate School of Science, The University of Tokyo, 7-3-1 Hongo, Bunkyo-ku, Tokyo 113-0033, Japan. ${ }^{2}$ Neutron Science and Technology Center, CROSS, 162-1 Shirakata, Tokai, Naka, Ibaraki 319-1106, Japan. ${ }^{3}$ Graduate Faculty of Interdisciplinary Research, University of Yamanashi, 4-3-11 Takeda, Kofu, Yamanashi 400-8511, Japan. ${ }^{4}$ Computational Engineering Applications Unit, RIKEN, 2-1 Hirosawa, Wako, Saitama 351-0198, Japan. ${ }^{5}$ JPARC Center, Japan Atomic Energy Agency, 2-4 Shirakata, Tokai, Naka, Ibaraki 319-1195, Japan. *email: kom@eqchem.s.u-tokyo.ac.jp
} 
W ater freezes below $0{ }^{\circ} \mathrm{C}$ at ambient pressure, ordinarily to ice $\mathrm{I}_{\mathrm{h}}$ with a hexagonal stacking sequence. However, it is also known to produce "ice $I_{c}$ " nominally with a cubic stacking sequence under certain conditions $s^{1}$, and its existence in Earth's atmosphere ${ }^{2-4}$, or in comets ${ }^{5,6}$ is debated. "Ice $\mathrm{I}_{c}$ ", or called as cubic ice, was first identified in 1943 by König 7 , who used electron microscopy to study the condensation of ice from water vapor to a cold substrate. Subsequently, many different routes to "ice $\mathrm{I}_{\mathrm{c}}$ " have been established, such as the dissociation of gas hydrates, warming amorphous ices or annealing high-pressure ices recovered at ambient pressure, freezing of $\mu$ - or nano-confined water (see ref. ${ }^{1}$ ). Despite the numerous studies on "ice $\mathrm{I}_{c}$ ", its structure has not been fully verified, because the diffraction patterns of "ice $I_{c}$ " show signatures of stacking disorder ${ }^{1,8,9}$, and ideal ice $I_{c}$ without stacking disorder had not been formed until very recently ${ }^{10}$. This is the reason why "ice $I_{c}$ " is double-quoted ${ }^{1}$, and it is recently proposed that the stackingdisordered ice should not be termed as ice $\mathrm{I}_{\mathrm{c}}$, but as ice $\mathrm{I}_{\mathrm{sd}}{ }^{8}$.

"Ice $I_{c}$ " (ice $I_{s d}$ ) is known as a metastable form of ice at atmospheric pressure. But, recent computer simulations suggest that even ice $I_{\text {sd }}$ could be the stable phase for crystallites up to sizes of at least 100,000 molecules $^{11}$. The stability of stackingdisordered ices is extremely important because of the ubiquitous nature of ice. Stacking-disordered ice can be characterized by the degree of ice cubicity, $\chi$, which is defined as the fraction of cubic stacking $1,8,9,12,13$. Until very recently, the highest cubicity was limited to $\sim 80 \% 8,14$, but it has been reported that ideal ice $\mathrm{I}_{\mathrm{c}}$ with $100 \%$ cubicity has been obtained by annealing ice XVII ${ }^{10}$.

Recently new ice polymorphs, ice XVI ${ }^{15}$ and ice XVII ${ }^{16,17}$ are obtained by degassing gas molecules from neon and hydrogen hydrates, respectively. From these findings, we hypothesized that ideal ice $\mathrm{I}_{c}$ could be obtained by degassing hydrogen from hydrogen hydrate, $\mathrm{C}_{2}$. Five different phases in the $\mathrm{H}_{2}-\mathrm{H}_{2} \mathrm{O}$ system have been reported to date (see ref. ${ }^{18}$ ): Among them, neutron diffraction experiments have never been conducted for the higher-pressure phases, $C_{1}$ and $C_{2}$, probably due to the technical difficulty in loading hydrogen into a pressure vessel, or compressing it to pressures in the giga-pascal range. To synthesize ideal ice $I_{c}$, decompression under low-temperature conditions for degassing is necessary, which is also not straight-forward using conventional pressure-temperature controlling systems. We have developed a Mito system ${ }^{19}$, and have overcome these technical difficulties (see details in Methods).

Here we present the neutron and X-ray diffraction results showing ice $I_{c}$ without stacking disorder, obtained from degassing hydrogen hydrate $\mathrm{C}_{2}$. We also report an unexpected amorphouslike state in the transformation from $\mathrm{C}_{2}$ to ice $\mathrm{I}_{\mathcal{c}}$, and the thermal stability of ice $\mathrm{I}_{\mathrm{c}}$.

\section{Results}

The route to obtain ice $\mathrm{I}_{\mathbf{c}}$. We started by using a mixture of $\mathrm{D}_{2} \mathrm{O}$ and $\mathrm{MgD}_{2}$, which is an internal deuterium source, to synthesize hydrogen hydrate, $\mathrm{C}_{2}$. After loading the mixture into a pressuretemperature controlling system, $\mathrm{MgD}_{2}$ was decomposed by heating at $403 \mathrm{~K}$ and at ca. $0 \mathrm{GPa}$ for $1 \mathrm{~h}$ through a nominal reaction of $\mathrm{MgD}_{2}+3 \mathrm{D}_{2} \mathrm{O} \rightarrow \mathrm{Mg}(\mathrm{OD})_{2}+2 \mathrm{D}_{2}+\mathrm{D}_{2} \mathrm{O}$ (at $b$ in Fig. 1, the observed neutron diffraction patterns are shown in Supplementary Fig. 1). Then, the samples were cooled to room temperature (at $c$ in Fig. 1) and typically compressed up to $\sim 3 \mathrm{GPa}$ until a $\mathrm{C}_{2}$ phase was observed (at $d$ in Fig. 1).

The neutron diffraction pattern for the $\mathrm{C}_{2}$ phase obtained at $3.3 \mathrm{GPa}$ and $300 \mathrm{~K}$ (at $d$ in Fig. 1) was analyzed by the Rietveld method. We adopted a splitting site model for guest $\mathrm{D}$ atoms located at the $48 f$ site $(x, 1 / 8,1 / 8)$, and the host structure was identical to ice $\mathrm{I}_{\mathrm{c}}{ }^{9}(F d \overline{3} m, \mathrm{O}$ at the $8 b$ site $(3 / 8,3 / 8,3 / 8), \mathrm{D}$ at the

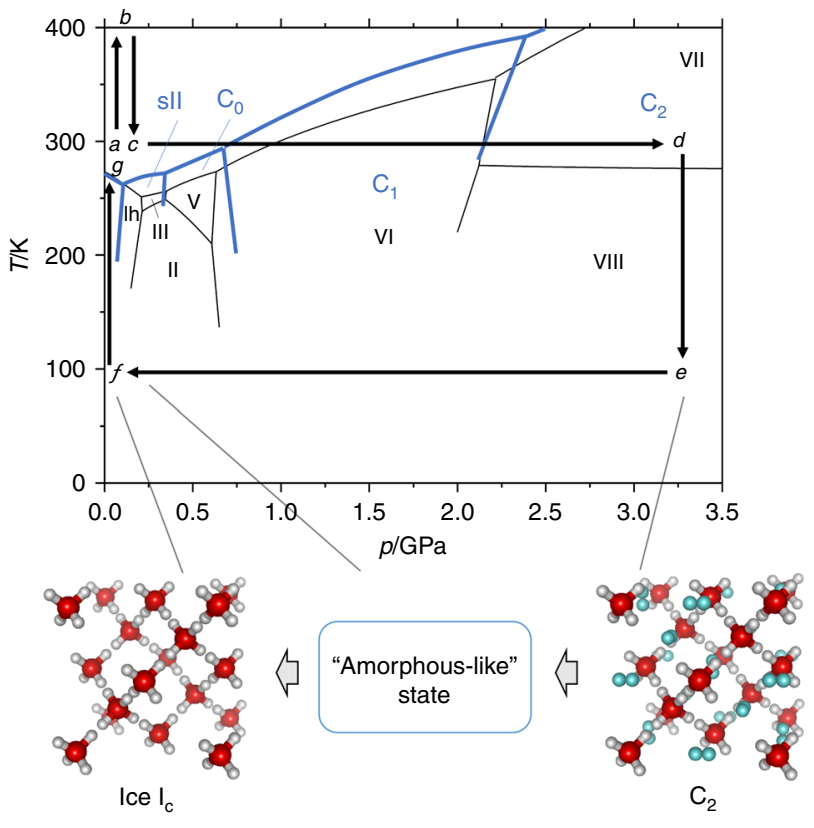

Fig. 1 Phase diagram of hydrogen hydrate and ice with experimental paths in this study. Phase boundaries for hydrogen hydrates and ices are drawn using thick blue lines and thin black lines, respectively. Experimental $p-T$ paths are shown as black arrows in alphabetical sequence from $a$ to $g$. The structural models for a high-pressure form of hydrogen hydrate, $C_{2}$, and ice $I_{c}$ are schematically drawn with a newly found amorphous-like state as an intermediate transitional state from $\mathrm{C}_{2}$ to ice $\mathrm{I}_{\mathrm{c}}$. Red, white, and light blue balls in the structure model depict oxygen, hydrogen in water molecules, and hydrogen in guest molecules, respectively. Note that hydrogens in water molecules are disordered, so that two of four possible sites surrounding one oxygen are actually occupied.

$32 e$ site $(x, x, x))$. The calculated diffraction pattern was in good agreement with the observed one, as shown in Fig. 2a. The refined structural parameters are listed in Supplementary Table 1.

The sample was then cooled from $300 \mathrm{~K}$ to $100 \mathrm{~K}$ at around $3 \mathrm{GPa}$ (path $d \rightarrow e$ ). In the diffraction pattern taken at $e$ in Fig. 1, peaks from solid deuterium (phase I) appeared at around $200 \mathrm{~K}$ (Supplementary Fig. 2), which is consistent with the known melting curve of hydrogen ${ }^{20}$. This observation indicates that fluid deuterium coexisted with $\mathrm{C}_{2}$ through the path from $b$ to $d$.

The $\mathrm{C}_{2}$ phase persisted at pressures at least as low as $0.5 \mathrm{GPa}$ on decompression at $100 \mathrm{~K}$ (path $e \rightarrow f$ ). However, surprisingly, the Bragg peaks of $\mathrm{C}_{2}$ mostly disappeared at $0.2 \mathrm{GPa}$ (Fig. 3). This phenomenon is totally unexpected, because the host structure of gas hydrates retains its framework in the previous cases with ice $\mathrm{XVI}^{15}$ and XVII ${ }^{16}$. The sample was further decompressed to $0 \mathrm{GPa}$ and evacuated using a turbo-molecular-pump. The broad peaks corresponding to ice $I_{c}$ appeared at this stage. The peak disappearance of $\mathrm{C}_{2}$ before the appearance of ice $\mathrm{I}_{c}$ was reproducibly observed in at least two separate neutron runs and one X-ray diffraction run for a hydrogenated sample (Supplementary Fig. 3). In the neutron diffraction pattern at $0.2 \mathrm{GPa}$, except for the Bragg peaks from $\mathrm{Mg}(\mathrm{OD})_{2}$, only a broad peak was observed at around $d=3.75 \AA$, which was between the peak positions of 111 of $C_{2}$ and that of Ice $I_{c}$ (Fig. 3). This fact implies that this state does not have long-range periodicity like a normal crystal, but has only local-ordering like an amorphous or nanocrystal. Considering the observed $d$-spacing, this amorphous-like form would be an intermediate transition state from $\mathrm{C}_{2}$ to ice $\mathrm{I}_{\mathcal{c}}$, which forms while hydrogen molecules are partially degassed. It is highly likely that this apparent amorphization is derived from the 


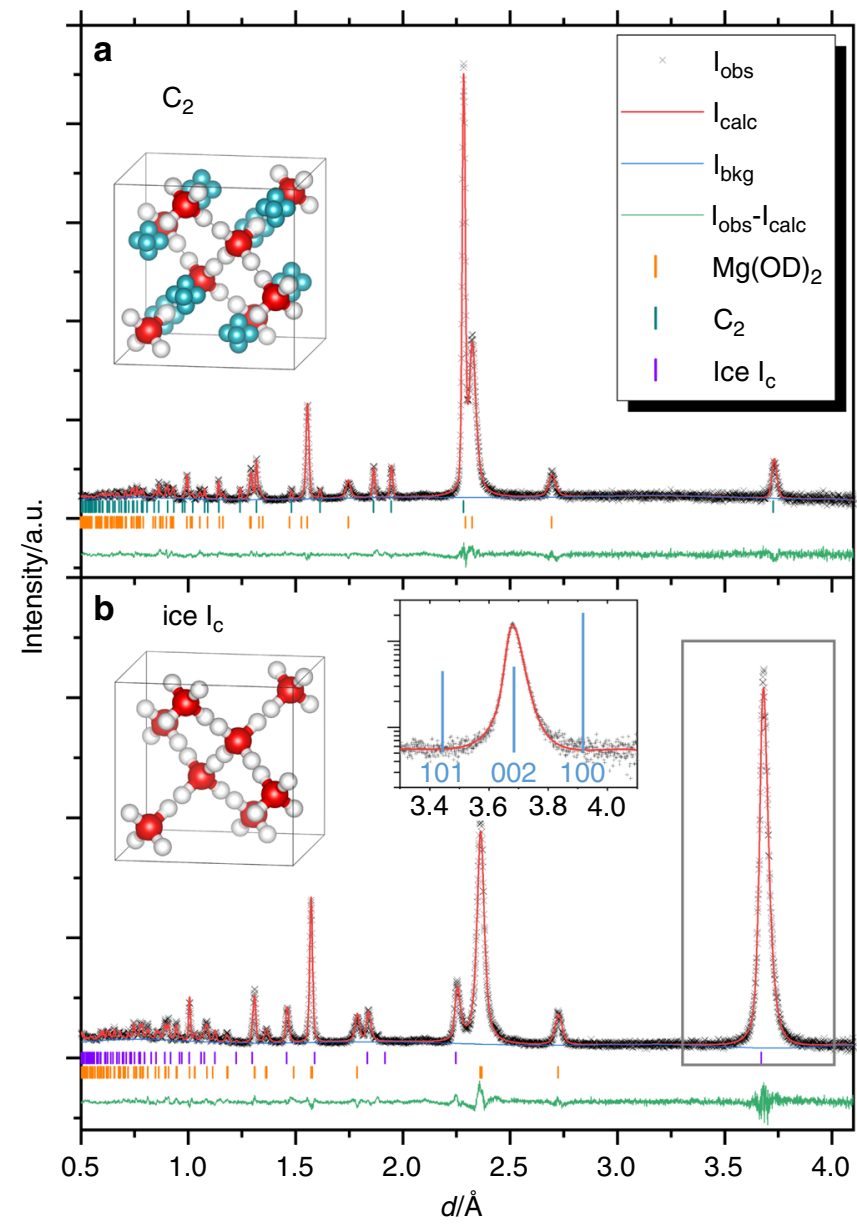

Fig. 2 Results of Rietveld analyses for neutron diffraction patterns. The patterns of $\mathbf{a}$ hydrogen hydrate, $\mathrm{C}_{2}$, and $\mathbf{b}$ ice $\mathrm{I}_{\mathrm{c}}$ were obtained at $3.3 \mathrm{GPa}$ and $300 \mathrm{~K}$ (at $d$ in Fig. 1), and at $0 \mathrm{GPa}$ and $130 \mathrm{~K}$ (in the path $f \rightarrow g$ ). The inset diffraction pattern in $\mathbf{b}$ shows the expanded area for 111 reflections, shown as a box in the main figure, with logarithmic scale. The calculated peak positions of ice $I_{h}$ are also shown as light blue lines with their indices in the inset. Structure models for $C_{2}$ and ice $I_{c}$ are also shown as insets in $\mathbf{a}$ and $\mathbf{b}$, respectively.

lattice mismatch between $C_{2}$ and ice $I_{c}$, originating from the relatively small cage in the host framework of the ice $\mathrm{I}_{\mathrm{c}}$ structure.

From the X-ray diffraction run, ice $\mathrm{I}_{\mathfrak{c}}$, which may partially include molecular hydrogen, even appeared at $0.1 \mathrm{GPa}$ through the transition from the $\mathrm{C}_{2}$ phase to the amorphous-like state, even under pressure (Supplementary Fig. 3). This also represents a difference from the previous cases of ice XVI and XVII; ice XVI is formed under evacuation ${ }^{15}$, and hydrogen molecules can be refilled into ice XVII at an order of 10 bar of pressure ${ }^{16}$. It is worth noting that the partially degassed states are allowed in the cases of both ice XVI and XVII, so that the guest molecules can be continuously degassed from a fully occupied state to an empty state. The observed phase-separation behavior even under pressure in the ice $\mathrm{H}_{2}-\mathrm{H}_{2} \mathrm{O}$ system indicates that the partially degassed $\mathrm{C}_{2}$ phase would be unstable compared to the fully occupied or emptied phases, probably due to their latticemismatch.

The Bragg peaks in the neutron diffraction pattern for ice $\mathrm{I}_{c}$ obtained at $100 \mathrm{~K}$ were still broad, probably due to the small crystallite size and/or the remaining guest hydrogen molecules. The peaks of ice $I_{c}$ sharpened with increasing temperature. This sharpening is dependent not only on temperature but also on time, which indicates that it is kinetic behavior.

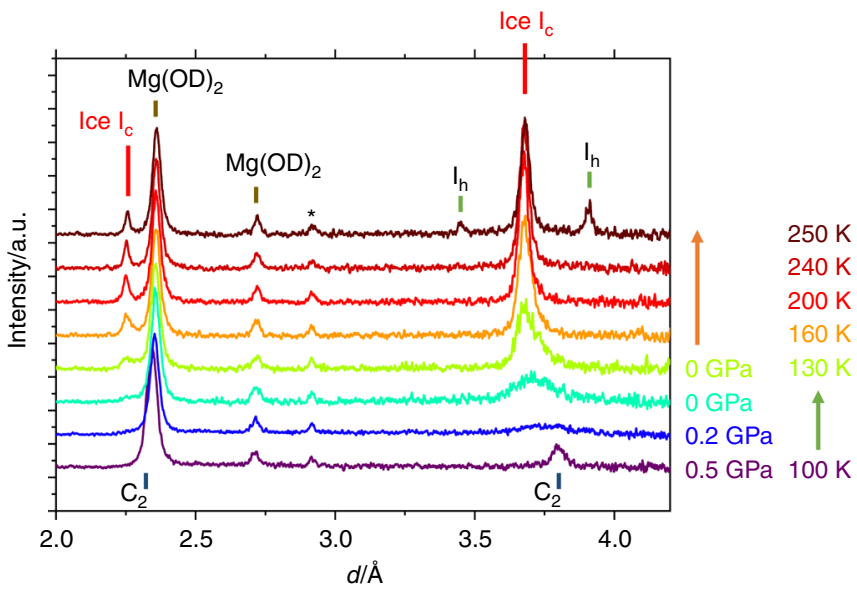

Fig. 3 Neutron diffraction patterns showing the transformation from $C_{2}$ to ice $\mathbf{I}_{\mathbf{c}}$. The patterns were obtained with decreasing pressure at $100 \mathrm{~K}$ (path $e \rightarrow f$ ) and with increasing temperature at $0 \mathrm{GPa}$ (path $f \rightarrow g$ ).

Corresponding temperatures and pressures are shown at the right side of the respective patterns, and the arrows mean that temperature or pressure kept constant. Most observed peaks are identified as $C_{2}$, ice $I_{c}, M g(O D)_{2}$, or ice $I_{h}$. The peak marked by an asterisk is a parasitic peak from the highpressure cell.

Structure refinement for ice $\mathbf{I}_{\boldsymbol{c}}$. We conducted a separate run in order to obtain a neutron diffraction pattern for the structure refinement of the ice $I_{c}$. In this run, the neutron diffraction pattern was obtained at $130 \mathrm{~K}$, which is well below the temperature at which the nucleation of ice $\mathrm{I}_{\mathrm{h}}$ occurs ${ }^{21}$. We confirmed that the peak width did not change in the temperature region from $130 \mathrm{~K}$ to $180 \mathrm{~K}$, such that the peak sharpening was almost complete, even at $130 \mathrm{~K}$. The obtained neutron diffraction pattern was well fitted using the ice $I_{c}$ structure model $^{9}$, as shown in Fig. $2 \mathrm{~b}$ and Supplementary Table 1 . We also conducted the Rietveld analysis using $\mathrm{C}_{2}$ structure model, and found that the occupancy of the D2 site is zero, within experimental error (occ $(D 2)=-0.001(1))$. This shows that the guest hydrogen molecules are below the detectable limit at $130 \mathrm{~K}$ under evacuation. The peak profile around 111 peak of ice $I_{c}$ has neither the feature of stacking disorder nor the peaks from ice $\mathrm{I}_{\mathrm{h}}$, as shown in the diffraction pattern in the region at around $d=3.9 \AA$, where the strongest 100 reflection of ice $I_{h}$ is expected (see inset in Fig. $2 b$, and more detailed discussion for the peak broadening for ice $I_{c}$ is described in Supplementary Note, Supplementary Table 2 and Supplementary Fig. 4). This should be a clear indication of the presence of ideal ice $I_{c}$ without stacking disorder $(\chi=100 \%)^{13}$, as clear as the recent discovery of ideal ice $I_{c}$ by annealing ice XVII ${ }^{10}$.

Thermal stability of ice $I_{c}$. It is also noteworthy that the ice $I_{c}$ surprisingly persists up to at least $240 \mathrm{~K}$ until ice $\mathrm{I}_{\mathrm{h}}$ started to appear at $250 \mathrm{~K}$ (Fig. 3). The temperature of $240 \mathrm{~K}$ corresponds to the upper limit of the reported metastable region of "ice $I_{c}$ " (ice $\left.\mathrm{I}_{\mathrm{sd}}\right)^{1}$. However, in stacking-disordered ice, the cubic stacking sequence starts to change into a hexagonal stacking sequence at a much lower temperature, and the phase transition to ice $\mathrm{I}_{\mathrm{h}}$ is completed at $240 \mathrm{~K}$. The notable stability of the ice $\mathrm{I}_{\mathrm{c}}$ would be derived from the lack of stacking disorder. The stackingdisordered ice has more dislocations, which promote the phase transformation from ice $I_{s d}$ to ice $I_{h}$ by reducing the activation energy required to change the stacking sequence ${ }^{22}$. This is also supported by a recent mesoscopic-size calculation ${ }^{23}$. Note here that the critical temperature of $240 \mathrm{~K}$ has been identified as the temperature above which ice $\mathrm{I}_{\mathrm{h}}$ without cubic stacking faults 
forms spontaneously, which is the reason for the anomalous selfpreservation regime of natural gas hydrates 24 .

The diffraction pattern observed at $250 \mathrm{~K}$ looks a mixture of bulk ice $\mathrm{I}_{\mathrm{c}}$ and $\mathrm{I}_{\mathrm{h}}$, rather than stacking-disordered ice with many stacking faults, judging from "stackogram" reported in the literatures 8,13 . At $250 \mathrm{~K}$, crystal growth would be dominant, rather than crystal nucleation. Therefore, once a crystallite nucleates, it quickly grows before other crystallites nucleate, resulting in the mixture of ice $I_{c}$ and $I_{h}$, rather than stackingdisordered ice. This observation also suggests a smaller number of dislocations in the ice $\mathrm{I}_{\mathrm{c}}$ observed in this study. On the contrary, the remarkable stability of the ice $I_{c}$ and the bulk mixture of ice $I_{c}$ and $\mathrm{I}_{\mathrm{h}}$ at $250 \mathrm{~K}$ strongly support the conclusion that the obtained ice is not stacking-disordered, and it can therefore be called ice $\mathrm{I}_{\mathrm{c}}$ without the need for quotation marks.

The discovery of ideal ice $I_{c}$ will allow us to research the real physical properties of ice $\mathrm{I}_{\mathrm{c}}$ without stacking disorder. For example, accurate heat capacity or vapor pressure measurements from low temperature will provide the free energy of ice $I_{c}$, which settle the long-standing argument for the thermodynamic stability of ice $I_{c}$ compared to ice $I_{h}$. The physical properties of the ideal ice $I_{c}$ are also important to understand how stacking disorder plays a role in the physical properties of ice $I_{s d}$. For instance, since the thermal conductivity of ice $I_{s d}$ is significantly smaller than ice $\mathrm{I}_{h}{ }^{25}$, the difference of thermal conductivities between ices $I_{h}$ and $I_{c}$ will emboss the effect of stacking disorder. It is also interesting what will be happened when ice $I_{c}$ is compressed under low temperature; whichever ice $I_{c}$ will be transformed into HDA (High Density Amorphous ice ${ }^{26}$ ) or not? In any case, it is worth conducting what we previously did for ice $I_{h}$, to this ideal ice $I_{c}$ as well.

\section{Methods}

Synthesis of $\mathbf{M g D}_{\mathbf{2}} \cdot \mathrm{MgD}_{2}$, used as the starting material in this study, was synthesized from reagent-grade $\mathrm{MgH}_{2}$ as follows. $\mathrm{MgH}_{2}$ powder (Wako pure chemical industries, Ltd.) was purchased and further ground in an agate motor to increase the surface area, after which it was placed in a copper tube with a diameter of $4 \mathrm{~mm}$ and a length of $40 \mathrm{~mm}$. The tube was mechanically sealed and but not welded, allowing the transfer of hydrogen gas. The copper tube was inserted into a $1 / 4$ " Inconel tube and connected in parallel to a deuterium gas cylinder and a turbomolecular pump (TMP) with 1/16" stainless tubes and stop bulbs. The Inconel tube, including the sample copper tube, was heated to $773 \mathrm{~K}$ for $1 \mathrm{~h}$ using a tube furnace under evacuation using the TMP. Under these conditions, $\mathrm{MgH}_{2} \mathrm{com}$ pletely decomposed to $\mathrm{Mg}$ and $\mathrm{H}_{2}{ }^{27}$, and the degassed $\mathrm{H}_{2}$ was evacuated. Then, the $\mathrm{D}_{2}$ gas was introduced up to $4 \mathrm{MPa}$, and the temperature was cycled at the rate of $1 \mathrm{~K} / \mathrm{min}$ between $673 \mathrm{~K}$ and $773 \mathrm{~K}$ while keeping the pressure at $4 \mathrm{MPa}$, which represents stable and unstable conditions for $\mathrm{MgH}_{2}{ }^{27}$, and this temperature cycle was repeated for 20 times. This activation process is necessary for the reaction $\mathrm{Mg}+\mathrm{D}_{2} \rightarrow \mathrm{MgD}_{2}$. Finally, the $p-T$ conditions were maintained at $673 \mathrm{~K}$ and 4 $\mathrm{MPa}$ for 3 days to complete the reaction. The recovered sample was analyzed by powder X-ray diffraction (MiniFlex-II, Rigaku) and identified to be $\mathrm{MgD}_{2}$ with a trace amount of $\mathrm{MgO}$. Both $\mathrm{MgD}_{2}$ and $\mathrm{MgO}$ react with $\mathrm{D}_{2} \mathrm{O}$ and produce $\mathrm{Mg}$ $(\mathrm{OD})_{2}$, so this small amount of $\mathrm{MgO}$ does not affect the conclusion of this study.

Neutron diffraction and $\boldsymbol{p}$-T control. Neutron powder diffraction experiments were conducted at the beamline PLANET ${ }^{28}$ in the Material and Life Science Experiment Facility (MLF) of J-PARC, Ibaraki, Japan. The incident beam consists of $25 \mathrm{~Hz}$ pulsed spallation neutrons produced from a liquid $\mathrm{Hg}$ target via a decoupled moderator and traveled through collimators, choppers and supermirror guides to the sample positioned at $25.0 \mathrm{~m}$ from the moderator ${ }^{28}$. Approx. $20 \mathrm{mg}$ of $\mathrm{MgD}_{2}$, synthesized as described above, was filled into TiZr null scattering gaskets, and $\mathrm{D}_{2} \mathrm{O}$ water $(99.9 \%$, Wako pure chemical industries, Ltd.) was dropped on the $\mathrm{MgD}_{2}$ powder, resulting in the molar ratio of $\mathrm{MgD}_{2}: \mathrm{D}_{2} \mathrm{O} \sim 1: 3$. The gaskets were sandwiched between a pair of tungsten carbide anvils, and loaded by using a hybrid Mito system, which is a modified version of an original pressure-temperature variable Mito system ${ }^{19}$. The hybrid Mito system uses both flowing liquid nitrogen and a $4 \mathrm{~K}$ cryostat (RDK-415D, Sumitomo Heavy Industries, Ltd.), which allows us to control temperature rapidly, owing to the large latent heat of liquid nitrogen and efficient thermal insulation by zirconia and GFRP seats. The hybrid Mito system also allows us to achieve temperatures below $77 \mathrm{~K}$, and reach a minimum temperature of $\sim 35 \mathrm{~K}$, owing to the cryostat. Another remarkable feature of the hybrid Mito system is that it affords pressure control, even at low temperature, as well as the original Mito system, which is indispensable for this study. Flexible copper cloths were attached on the support rings of the anvils, and the cloths were placed in contact with the cold head of the cryostat for thermal conduction. The accessible minimum temperature of the hybrid Mito system is $\sim 35 \mathrm{~K}$, which may be the current technical limitation due to an unavoidable influx of heat from the surrounding cell. The sample pressure was estimated from the observed lattice parameter of $\mathrm{Mg}(\mathrm{OD})_{2}$ brucite using the equation of states ${ }^{29}$ and the observed unit cell volume of brucite at $0 \mathrm{GPa}$, assuming the temperature derivative of the bulk modulus of brucite, $d K / d T$, was $\sim 0$. Although this assumption may cause some error in the pressure estimated at low temperature, we placed emphasis on avoiding unwanted Bragg peaks from additional sources of pressure marker. Moreover, the error would be too small to affect the conclusion. The sample position was aligned by scanning to maximize the sample scattering intensity. The obtained intensities from the sample in the cell was subtracted by the intensity of the empty cell, and subsequently normalized by the attenuation corrected intensity of vanadium pellet in the cell, which was also subtracted by the intensity of the empty cell ${ }^{30}$. The Rietveld analyses were performed using the GSAS ${ }^{31}$ with EXPGUI ${ }^{32}$, and the crystal structure was drawn with the VESTA program ${ }^{33}$. The GSAS TOF profile function $3^{31}$ is used as the profile function in the Rietveld analyses.

X-ray diffraction. Powder X-ray diffraction measurements using a $\mathrm{H}_{2} \mathrm{O}$ (Milli-Q) and $\mathrm{MgH}_{2}$ (Wako pure chemical industries, Ltd.) mixture as starting materials were performed at the beamline BL-18C in the Photon Factory (KEK, Tsukuba, Japan). Samples were exposed to $0.6134 \AA$ monochromatized synchrotron radiation, and the diffracted scattering was detected by an imaging plate (IP). The pressure was generated using $\mathrm{CuBe}$ alloy diamond-anvil cells and the temperature was controlled using a $4 \mathrm{~K}$ GM cryostat (MiniStat, Iwatani Co.) equipped with a temperature controller (Model 335, Lakeshore). Sample pressure was estimated from the difference in the R1 line wavelengths of rubies inside and outside the sample chamber ${ }^{34}$. The temperature was monitored using a Si-diode sensor inserted in the cold head edge. We confirmed that the measured temperature was almost the same as that of the diamond anvils after temperature stabilization. The experimental $p-T$ path was basically identical to the case of neutron diffraction, as shown in Fig. 1, while the achieved pressure at path $d$ was $4.1 \mathrm{GPa}$.

One conically shaped Boehler-Almax type diamond-anvil ${ }^{35}$ with a $0.6 \mathrm{~mm}$ culet was placed in the direction of the detector with an opening angle of $2 \theta<40^{\circ}$, whereas a conventional anvil with a $0.8 \mathrm{~mm}$ culet was positioned in the direction of the X-ray source. A CuBe plate with a hole of diameter $0.3 \mathrm{~mm}$ and an initial thickness of $0.2 \mathrm{~mm}$ was used as a gasket. This gasket was not subjected to preindentation. The load was applied by driving the piston by bellows using a He gas cylinder. The bellows allow us to control pressure at a few kbar more precisely than conventionally used membranes.

DFT calculations. Quantum Espresso ${ }^{36}$ was used for the DFT calculations ${ }^{37,38}$. We used Perdew-Burke-Ernzerhof (so-called PBE) type nonempirical exchangecorrelation functions ${ }^{39}$ for this study. The pseudopotentials were derived using projector augmented-wave approximation ${ }^{40}$. The dispersion effects were taken into account using the exchange-hole dipole moment method (XDM), which calculates coefficients for polynomial of DFT-D dispersion energy ${ }^{41}$ from the exchange-hole dipole moment calculated from simulated electron wave function ${ }^{42,43}$. XDM damping function parameters are taken from ${ }^{44}$. The enthalpies of four possible configurations for the ordered form of ice $\mathrm{I}_{\mathrm{c}}$ were calculated within a unit cell with a kinetic energy cutoff of $70 \mathrm{Ry}$ and a Brillouin zone $\mathrm{k}$ mesh of $8 \times 8 \times 8$. The cell parameters and atomic coordinates were optimized using BFGS quasiNewtonian methods at atmospheric pressure.

\section{Data availability}

The primary data that support the plots within this paper and other finding of this study are available from the corresponding author on reasonable request. The neutron crystallographic coordinates for structures reported in this study have been deposited at the Cambridge Crystallographic Data Centre (CCDC), under deposition numbers 1973757, 1973759. These data can be obtained free of charge from The Cambridge Crystallographic Data Centre via www.ccdc.cam.ac.uk/data_request/cif.

Received: 23 September 2019; Accepted: 3 January 2020; Published online: 03 February 2020

\section{References}

1. Kuhs, W. F., Sippel, C., Falenty, A. \& Hansen, T. C. Extent and relevance of stacking disorder in “ice I". Proc. Natl Acad. Sci. USA 109, 21259-21264 (2012).

2. Murphy, D. M. Dehydration in cold clouds is enhanced by a transition from cubic to hexagonal ice. Geophys. Res. Lett. 30, 2230 (2003).

3. Riikonen, M. et al. Halo observations provide evidence of airborne cubic ice in the Earth's atmosphere. Appl. Opt. 39, 6080-6085 (2000).

4. Whalley, E. Scheiner's halo: evidence for ice Ic in the atmosphere. Science 211 389-390 (1981). 
5. Gronkowski, P. The search for a cometary outbursts mechanism: a comparison of various theories. Astronomische Nachrichten: Astronomical Notes 328, 126-136 (2007).

6. Prialnik, D. \& Bar-Nun, A. Crystallization of amorphous ice as the cause of comet P/Halley's outburst at 14 AU. Astron. Astrophys. 258, L9-L12 (1992).

7. König, H. Eine kubische Eismodifikation. Z. Kristallogr. Crsyt. Mater. 105, 279 (1943).

8. Malkin, T. L. et al. Stacking disorder in ice I. Phys. Chem. Chem. Phys. 17, 60-76 (2015)

9. Kuhs, W. F., Bliss, D. V. \& Finney, J. L. High-resolution neutron powder dffraction study of ice-Ic. J. Phys. 48, 631-636 (1987).

10. del Rosso, L. et al. Cubic ice Ic without stacking defects obtained from ice XVII, Nat. Mater. https://doi.org/10.1038/s41563-020-0606-y (2020).

11. Lupi, L. et al. Role of stacking disorder in ice nucleation. Nature 551, 218 (2017).

12. Malkin, T. L., Murray, B. J., Brukhno, A. V., Anwar, J. \& Salzmann, C. G. Structure of ice crystallized from supercooled water. Proc. Natl Acad. Sci. USA 109, 1041-1045 (2012)

13. Hansen, T. C., Sippel, C. \& Kuhs, W. F. Approximations to the full description of stacking disorder in ice I for powder diffraction. Z. f.ür. Kristallographie 230, 75-86 (2015)

14. Amaya, A. J. et al. How Cubic Can Ice Be? J. Phys. Chem. Lett. 8, 3216-3222 (2017).

15. Falenty, A., Hansen, T. C. \& Kuhs, W. F. Formation and properties of ice XVI obtained by emptying a type sII clathrate hydrate. Nature 516, 231-233 (2014).

16. del Rosso, L., Celli, M. \& Ulivi, L. New porous water ice metastable at atmospheric pressure obtained by emptying a hydrogen-filled ice. Nat. Commun. 7, 13394 (2016).

17. del Rosso, L. et al. Refined structure of metastable ice XVII from neutron diffraction measurements. J. Phys. Chem. C. 120, 26955-26959 (2016).

18. Donnelly, M. E., Teeratchanan, P., Bull, C. L., Hermann, A. \& Loveday, J. S. Ostwald's rule of stages and metastable transitions in the hydrogen-water system at high pressure. Phys. Chem. Chem. Phys. 20, 26853-26858 (2018).

19. Komatsu, K. et al. Development of a new P-T controlling system for neutronscattering experiments. High. Press. Res. 33, 208-213 (2013).

20. Diatschenko, V. et al. Melting curves of molecular hydrogen and molecular deuterium under high pressures between 20 and 373 K. Phys. Rev. B 32, 381-389 (1985).

21. Hansen, T. C., Koza, M. M., Lindner, P. \& Kuhs, W. F. Formation and annealing of cubic ice: II. Kinetic study. J. Phys.: Condens. Matter 20, 285105 (2008).

22. Hondoh, T. Dislocation mechanism for transformation between cubic ice $I_{c}$ and hexagonal ice $\mathrm{I}_{\mathrm{h}}$. Philos. Mag. 95, 3590-3620 (2015).

23. Chan, H. et al. Machine learning coarse grained models for water. Nat. Commun. 10, 379 (2019).

24. Kuhs, W. F., Genov, G., Staykova, D. K. \& Hansen, T. Ice perfection and onset of anomalous preservation of gas hydrates. Phys. Chem. Chem. Phys. 6, 4917-4920 (2004).

25. Johari, G. P. \& Andersson, O. Effects of stacking disorder on thermal conductivity of cubic ice. J. Chem. Phys. 143, 054505 (2015).

26. Mishima, O., Calvert, L. D. \& Whalley, E. 'Melting ice' I at $77 \mathrm{~K}$ and $10 \mathrm{kbar}$ : a new method of making amorphous solids. Nature 310, 393-395 (1984).

27. Crivello, J.-C. et al. Review of magnesium hydride-based materials: development and optimisation. Appl. Phys. A 122, 97 (2016).

28. Hattori, T. et al. Design and performance of high-pressure PLANET beamline at pulsed neutron source at J-PARC. Nucl. Instr. Meth. Phys. Res. A 780, 55-67 (2015).

29. Horita, J., dos Santos, A. M., Tulk, C. A., Chakoumakos, B. C. \& Polyakov, V. B. High-pressure neutron diffraction study on $\mathrm{H}-\mathrm{D}$ isotope effects in brucite. Phys. Chem. Miner. 37, 741-749 (2010).

30. Komatsu, K. et al. Crystal structure of magnesium dichloride decahydrate determined by X-ray and neutron diffraction under high pressure. Acta Crystallogr. B 71, 74-80 (2015).

31. Larson, A. \& Von Dreele, R. General Structure Analysis System (GSAS). Los Alamos National Laboratory, Report LAUR-86-748 (2004).

32. Toby, B. EXPGUI, a graphical user interface for GSAS. J. Appl. Crystallogr. 34, 210-213 (2001).

33. Momma, K. \& Izumi, F. VESTA 3 for three-dimensional visualization of crystal, volumetric and morphology data. J. Appl. Crystallogr. 44, 1272-1276 (2011).

34. Piermarini, G. J., Block, S., Barnett, J. D. \& Forman, R. A. Calibration of the pressure dependence of the $\mathrm{R}_{1}$ ruby fluorescence line to $195 \mathrm{kbar}$. J. Appl. Phys. 46, 2774-2780 (1975).

35. Boehler, R. \& De Hantsetters, K. New anvil designs in diamond-cells. High. Press. Res. 24, 391-396 (2004).
36. Paolo, G. et al. QUANTUM ESPRESSO: a modular and open-source software project for quantum simulations of materials. J. Phys. Condens. Matter 21, 395502 (2009).

37. Hohenberg, P. \& Kohn, W. Inhomogeneous electron gas. Phys. Rev. 136, B864-B871 (1964).

38. Kohn, W. \& Sham, L. J. Self-consistent equations including exchange and correlation effects. Phys. Rev. 140, A1133-A1138 (1965).

39. Perdew, J. P., Burke, K. \& Ernzerhof, M. Generalized gradient approximation made simple. Phys. Rev. Lett. 77, 3865-3868 (1996).

40. Kresse, G. \& Joubert, D. From ultrasoft pseudopotentials to the projector augmented-wave method. Phys. Rev. B 59, 1758-1775 (1999).

41. Grimme, S., Antony, J., Ehrlich, S. \& Krieg, H. A consistent and accurate ab initio parametrization of density functional dispersion correction (DFT-D) for the 94 elements H-Pu. J. Chem. Phys. 132, 154104 (2010).

42. Becke, A. D. \& Johnson, E. R. Exchange-hole dipole moment and the dispersion interaction. J. Chem. Phys. 122, 154104 (2005).

43. Becke, A. D. \& Johnson, E. R. Exchange-hole dipole moment and the dispersion interaction revisited. J. Chem. Phys. 127, 154108 (2007).

44. Otero-de-la-Roza, A. \& Johnson, E. R. Van der Waals interactions in solids using the exchange-hole dipole moment model. J. Chem. Phys. 136, 174109 (2012).

\section{Acknowledgements}

We are grateful to Drs. J. Abe and K. Funakoshi for their assistance with the experiments, and to anonymous reviewers for their valuable comments, particularly on the relation with anomalous preservation of gas hydrates. Neutron diffraction experiments were performed through the J-PARC user programs (Nos. 2014B0187, 2015A0033, 2017A0092, 2017B0031). X-ray diffraction experiments were performed under the approval of the Photon Factory Program Advisory Committee (Proposal Nos. 2016G107, 2018G656). This study was supported by JSPS KAKENHI (Grant Numbers: 18H05224, 18H01936, 15H05829)

\section{Author contributions}

K.K. and S.M. conceived and designed the experiments. K.K., S.M., T.H., A.S-F., R.Y., K.Y., and H.K. conducted the neutron diffraction experiments. K.K., R.Y., K.Y., and H.K. conducted the X-ray diffraction experiments. F.N. carried out the DFT calculations and wrote the corresponding part of the manuscript. K.K. analyzed the data and wrote the manuscript with contributions from all authors.

\section{Competing interests}

The authors declare no competing interests.

\section{Additional information}

Supplementary information is available for this paper at https://doi.org/10.1038/s41467 020-14346-5.

Correspondence and requests for materials should be addressed to K.K.

Peer review information Nature Communications thanks John Loveday and the anonymous reviewer(s) for their contribution to the peer review of this work.

Reprints and permission information is available at http://www.nature.com/reprints

Publisher's note Springer Nature remains neutral with regard to jurisdictional claims in published maps and institutional affiliations.

Open Access This article is licensed under a Creative Commons Attribution 4.0 International License, which permits use, sharing, adaptation, distribution and reproduction in any medium or format, as long as you give appropriate credit to the original author(s) and the source, provide a link to the Creative Commons license, and indicate if changes were made. The images or other third party material in this article are included in the article's Creative Commons license, unless indicated otherwise in a credit line to the material. If material is not included in the article's Creative Commons license and your intended use is not permitted by statutory regulation or exceeds the permitted use, you will need to obtain permission directly from the copyright holder. To view a copy of this license, visit http://creativecommons.org/ licenses/by/4.0/

(C) The Author(s) 2020 